Reprint Series

17 January 1997 , Volume 275 , pp. 375-377

\title{
Depletion of the Outer Asteroid Belt
}

\author{
Jer-Chyi Liou* and Renu Malhotra
}




\title{
Depletion of the Outer Asteroid Belt
} Jer-Chyi Liou* and Renu Malhotra

\begin{abstract}
During the early history of the solar system, it is likely that the outer planets changed their distance from the sun, and hence, their influence on the asteroid belt evolved with time. The gravitational influence of Jupiter and Saturn on the orbital evolution of asteroids in the outer asteroid belt was calculated. The results show that the sweeping of mean motion resonances associated with planetary migration efficiently destabilizes orbits in the outer asteroid belt on a time scale of 10 million years. This mechanism provides an explanation for the observed depletion of asteroids in that region.
\end{abstract}

Asteroids are small, rocky bodies less than $1000 \mathrm{~km}$ in diameter that lie between the orbits of Mars and Jupiter in the region traditionally called the asteroid belt. The outer asteroid belt, from 3 to 5 astronomical units (AU) from the sun, is nonuniform and depleted of asteroids. The orbital eccentricities and inclinations, as functions of semi-

J.-C. Liou, SN3, NASA Johnson Space Center, Houston, TX77058, USA

R. Malhotra, Lunar and Planetary Institute, Houston, TX 77058. USA.

"To whom correspondence should be addressed. E-mail: liou@sn3.jsc.nasa.gov major axes, of 7100 numbered asteroids (1)

(Fig. 1) show four major features: (i) a lack of asteroids in the $1: 2$ interior mean motion resonance (MMR) (2) with Jupiter centered at $3.28 \mathrm{AU}$, (ii) a lack of asteroids between 3.5 and $3.9 \mathrm{AU}$, (iii) a concentration of asteroids in the $2: 3$ interior MMR with Jupiter centered at $3.97 \mathrm{AU}$, and (iv) a lack of asteroids beyond the 2:3 interior MMR.

The "gravitational hypothesis" (3) postulates that the gravitational forces of the planets are responsible for shaping the asteroid belt. This hypothesis has explained 
gaps at the $1: 3$ and $1: 2$ interior MMR locations and the depletion beyond the $2: 3$ interior MMR (4-6). However, recent numerical simulations of the solar system for a period of 1 billion years based on this hypothesis have failed to reproduce the observed lack of asteroids between 3.5 and 3.9 $\mathrm{AU}$, prompting the conjecture that this feature may he related to the distrihution of asteroids at the end of planetary formation or to other nongravitational processes (7, 8). Here we consider the planet migration hypothesis that hals heen invoked for several outer solar system problems $(9,10)$.

During the early history of the solar system, it is likely that gravitational scattering of planetesimals by giant planets caused the orhits of Saturn, Uranus, and Neptune to migrate outward and the orhit of Jupiter to migrate inward (11). This dynamical process, if it occurred, would explain the peculiar orhit of Pluto and the capture of Kuiper Belt objects in MMRs with Neptune $(9,10)$. The migration of the giant planets would also have affected the evolution of the main belt asteroids and may have been responsible for some of the hitherto unsolved mysteries of the ohserved structure of the asteroid helt (9). We numerically calculated the orbital evolution of 200 test asteroids initially in near-circular, low-inclination orbits in the outer asteroid belt under the perturbing forces of Jupiter and Saturn - when the planets are in their current orhits and when they are in radial migration-to determine how planetary migration would have affected the asteroid distribution.

We used two numerical integration methods: an implicit Runge-Kutta integrator with a self-adjusting step-size control (RADAU) (12) and a modified mixed-variable quasisymplectic mapping (13). RAI)AU handles close encounters between a test asteroid and a planet accurately, hut its slow calculation speed makes it impractical for a systematic study of the evolution of a large number of asteroids over 10 million years. The mapping method is at least one order of magnitude faster than RADAU, so it is suitable for studying the long-term evolution of test asteroids; however, it is not accurate for close encounters, so we terminated the integration of any asteroid that had a close encounter with any planet (14) and presumed that it had been removed from the asteroid population. We compared the results of simulations using both integrators for integration times less than 10 million years and found that, statistically, hoth integrators gave the same results. For integrations longer than 10 million yeats, we used only the mapping integrator.

We modeled the migration of a planet by applying a force opposite to (or along) its orhital velocity vector such that it migrated radially inward (or outward) over time. We included two planets in our simulations: Jupi- ter with a $0.2-\mathrm{AU}$ inward migration and Saturn with a 0.8 -AU ourward migration. We used two migration schemes: (i) a linear migration, wherein the planetary semimajor axes changed linearly with time, and (ii) an exponential migration, wherein the rates of change of planetary semimajor axes decayed exponentially with time, with migration time scales ranging from 1 million to 10 million years. The total integration times ranged from 1 million to 100 million years. Hundreds of test asteroids were initially placed between 3.2 and $4.2 \mathrm{AU}$. Their initial orbital eccentricities and inclinations were randomly chosen between 0 and 0.1 and between $0^{\circ}$ and $6^{\circ}$, respectively. Their initial longitudes of ascending node, longitudes of pericenter, and mean longitudes were all randomly chosen between $0^{\circ}$ and $360^{\circ}$. Once the initial conditions of test asteroids and planets were set up, their equations of motion were numerically integrated. In our calculations, the planets fully interacted with each other and acted on test asteroids, whereas the test asteroids did not affect the motions of planets.

Our simulation with no planet migration (Fig. 2B) is consistent with previous work: (i) most asteroids at the 1:2 interior MMR were depleted (5), (ii) asteroids at the $2: 3$ interior MMR were maintained in stable resonance, (iii) asteroids outside 4 AU were removed by close encounters with Jupiter (6), and (iv) the depletion of asteroids hetween 3.5 and 3.9 AU was only about $50 \%$ (7). The latter depletion fraction did not increase even with a 10 -fold longer integration of 1 billion years (8). On the other hand, in our simulations with planetary migration, the orbital migration of giant plan-

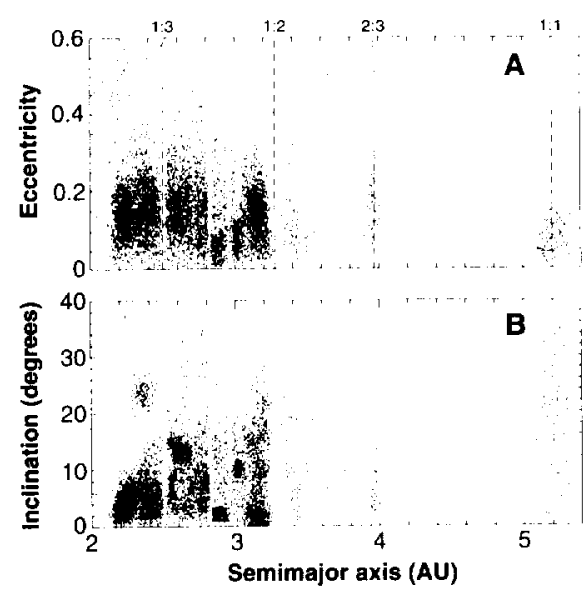

Fig. 1. Distribution of the observed asteroids between 2 and $5.4 \mathrm{AU}$ on the basis of their orbital eccentricities (A) and inclinations (B) as functions of semimajor axes. The gaps and concentrations of asteroids are associated with the mean motion resonances with Jupiter. The locations of four of them-1:3, 1:2, 2:3, and 1:1-are shown by dashed lines in (A). ets produced an efficient depletion in the region between 3.5 and $3.9 \mathrm{AU}$ on millionyear time scales (Fig. 2, C through E). The linear and exponential migration simulations gave similar results. They also indicate that the slower the planets migrate, the more efficient the depletion is.

Planet migration increased the efficienc) of depletion of asteroids in this particular region because the depletion was caused by the sweeping of MMRs through the region a: Jupiter migrated inward. It has been showr recently that, in the current planetary cun. figuration, astercids at the $4: 7,3: 5$, and $5: \varepsilon$ interior MMRs with Jupiter (located at $3.58,3.70$, and $3.80 \mathrm{AU}$, respectively) : Ir unstable over million-year time scales $(8$ 15). The orbit of an asteroid in one of those three resonances is highly chaotic: An ini tially circular orbit at these resonances wil have its orbital eccentricity pumped up in : short period of time; eventually its orbi crosses that of Jupiter, and close encounter with the planet remove it from that region lt is these three MMRs that provide the $50 \%$ depletion of asteroids from the oute

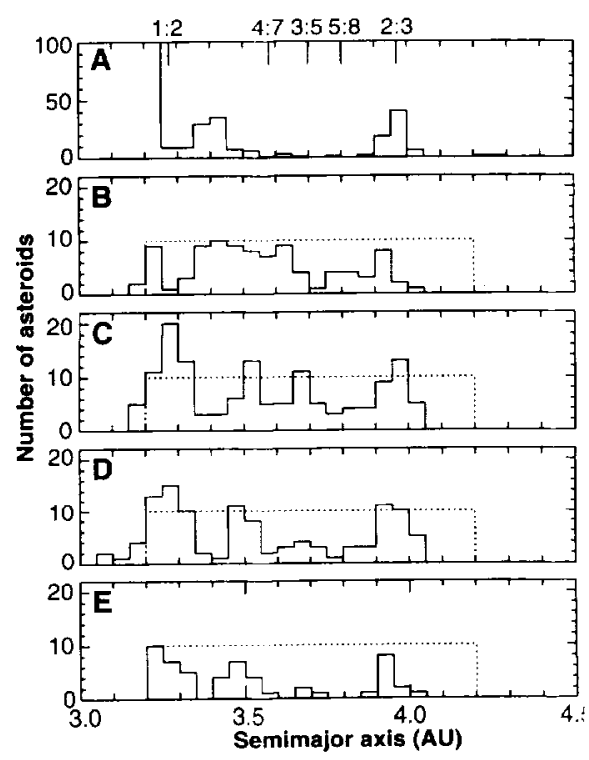

Fig. 2. (A) Distribution of the observed numbere asteroids. The locations of the $1: 2,4: 7,3: 5,5: \varepsilon$ and 2:3 interior MMRs are labeled by the boid lin segments at the top. (B) Distribution of 200 test as teroids after 100 million years of numerical simulatio in a sun-Jupiter-Saturn-asteroids system [adapte from (16)]. Jupiter and Saturn are interacting wit each other with no radial migration. The dotted lir indicates the initial distribution of the test astercid: The 1:2 gap was formed after 10 million years. Abor $50 \%$ of the original asteroids still remained betwee 3.5 and 3.9 AU at the end of the simulation. (C). (D and $(\mathbf{E})$ show results from three different numeric simulations of sun-Jupiter-Saturn-asteroids syster in which Jupiter migrated inward while Saturn $m$ grated outward linearly with time for 1,2 , and million years, respectively. Simulations with expr nential migration gave similar results. 
belt in the classical case (no planet migration). When the migration of giant planets is included or, more specifically, when the inward migration of Jupiter is included, those three resonances sweep through the region between 3.5 and $3.9 \mathrm{AU}$. Asteroids originally not in any resonances encounter those MMRs and get captured, and their orbits become chaotic (Fig. 3). It is this resonance-sweeping mechanism that depletes asteroids from the outer belt.

We also considered the effect of this mechanism on other parts of the asteroid belt. Asteroids outside $4 \mathrm{AU}$ were removed, whereas asteroids were maintained in the $2: 3$ interior MMR at 3.97 $\mathrm{AU}$ (Fig. 2), consistent with the observed asteroid distribution. However, in our simulations, asteroids were captured and maintained in the $1: 2$ interior MMR at $3.28 \mathrm{AU}$, contrary to the observed gap at this location. A plausible explanation for this deficiency is the time scale of instability for asteroids in that resonance. In the classical case (no planet migration), the time scale to clear the $1: 2$ gap is on the order of 10 million years $(5,16)$. A hint of this long-term effect is evident in Fig. 2E and is more obvious

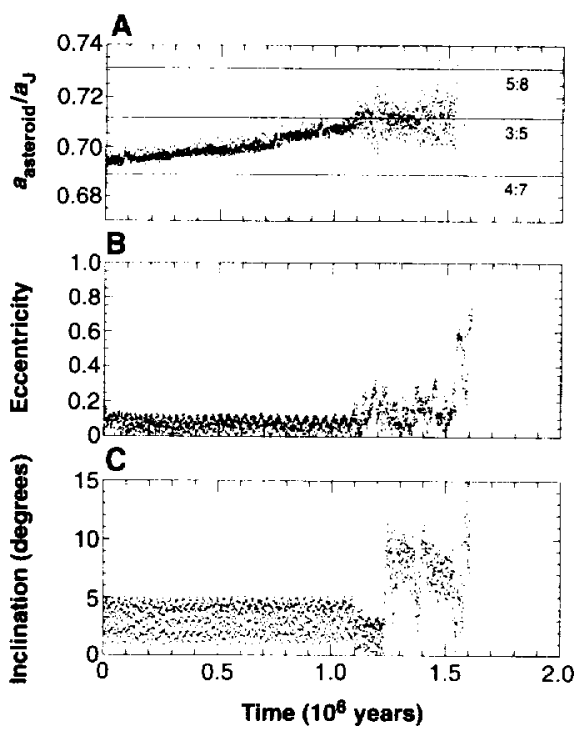

Fig. 3. Evolution of an asteroid in a sun-JupiterSaturn-asteroid system. The planetary migration time is 2 million years. (A) Semimajor axis of the test asteroid normalized to the semimajor axis of Jupiter. Three horizontal lines show the locations of the $5: 8,3: 5$, and $4: 7$ interior MMRs. As Jupiter migrated inward, the relative semimajor axis of the asteroid increased. This test asteroid was initially not in any MMR with Jupiter; however, the inward motion of Jupiter caused the 3:5 interior MMR to sweep by and capture this asteroid into resonance at 1.1 million years. The eccentricity $(\mathbf{B})$ and inclination (C) of the asteroid varied irregularly when it was in resonance. Finally, its eccentricity increased to such an extent that it began to cross the orbit of Jupiter, and subsequent close encounters with the giant planet finally made its orbit hyperbolic and removed it from the outer belt. in Fig. 2B. We conclude that the asteroids at the $1: 2$ interior MMR were removed by the long-term perturbations of the planets after planet migration ceased (17).

\section{REFERENCES AND NOTES}

1. E. Bowell, Asteroid Orbital Elements Database (Lowell Observatory, Flagstaff, AZ, 1996).

2. S. F. Dermott and C. D. Murray, Nature 301, 201 (1983). AMMR occurs when the orbital period of an asteroid is a simple multiple of Jupiter's orbital period. In addition to $1: 2$ and $2: 3$, interior MMRs are also associated with other gaps in the asteroid belt.

3. R. Greenberg and H. Scholl, in Asteroids, T. Gehrels, Ed. (Univ. of Arizona Press, Tucson, 1979), pp. 310333; A. M. Nobili, in Asteroids II, R. P. Binzel, T. Gehrels, M. S. Matthews, Eds. (Univ. of Arizona Press, Tucson, 1989), pp. 862-879.

4. J. Wisdom, Icarus 72, 241 (1987).

5. F. A. Franklin, Astron. J. 112, 1247 (1996); A. Morbidelli, ibid. 111, 2453 (1996).

6. B. Gladman and M. Duncan, ibid. 100, $1680(1990)$.

7. M. Lecar, F. Franklin, P. Soper, Icarus 96, 234 (1992).

8. M. Duncan, in Circumstellar Dust Disks and Planet Formation, R. Ferlet and A. Vidal-Madjar, Eds. (Editions Frontieres, Gif-sur-Yvette, 1994), pp. 245-255.

9. R. Malhotra, Nature 365, 819 (1993).

10. Astron. J. 110, 420 (1995).

11. J. A. Fernández and W.-H. Ip, lcarus 58, 109 (1984); Planet. Space Sci. 44, 431 (1996).

12. E. Everhart, in Dynamics of Comets: Their Origin and Evolution, A. Carusi and G. B. Valsecchi, Eds. (Rei- del, Boston, 1985), pp. 185-202.

13. R. Malhotra, Celestial Mech. Dyn. Astron. 60, 373 (1994)

14. Integration of a test asteroid was stopped, and the asteroid was presumed to have been removed from the population, if it came within 1 Hill radius $r_{H}$ of a planet. Once an object is less than $1 r_{H}$ from a planet, the dominant perturber is the planet, not the sun. The Hill radii of Jupiter and Saturn are 0.36 and $0.44 \mathrm{AU}$, respectively.

15. M. J. Hoiman and N. W. Murray, Astron. J. 112. 1278 (1996)

16. R. Malhotra, unpublished material.

17. Because of the geometric locations of the $4: 7,3: 5$, and 5:8 interior MMRs, Jupiter has to migrate inward, by about $0.2 \mathrm{AU}$, to remove asteroids in the 3.5- to 3.9-AU region. However, the migration time scale and the migration scheme (linear, exponential, or other) are less constrained on the basis of the population of asteroids in this region. The long-term dynamics of the asteroids at $2: 3$ and $1: 2$ interior MMRs after the planet migration ceased may provide more insight into these questions.

18. We thank H. Zook for enthusiastic discussions. This research was performed while J.-C.L. held a National Research Council-NASA Johnson Space Center Research Associateship and R.M. was a staff scientist at the Lunar and Planetary Institute (LPI), which is operated by the Universities Space Research Association under contract NASW-4574 with NASA. Support was also provided by NASA's Origins of Solar Systems Research Program under grant 4474 . This paper is LPI contribution 905.

4 September 1996; accepted 15 November 1996 